\title{
Tight focus of light using a micropolarizer and a microlens
}

\author{
Sergey S. Stafeev,,${ }^{1,2,}$ Liam O’Faolain, ${ }^{3}$ Victor V. Kotlyar, ${ }^{1,2}$ Anton G. Nalimov, ${ }^{1,2}$ \\ ${ }^{1}$ Image Processing Systems Institute, Russian Academy of Sciences, 151 Molodogvardeyskaya st., 443001, Samara, Russia \\ ${ }^{2}$ Samara State Aerospace University, 34 Moskovskoe shosse, 443086, Samara, Russia \\ ${ }^{3}$ SUPA, School of Physics and Astronomy of the University of St. Andrews, North Haugh, St. Andrews, KY16 9SS, Scotland, \\ $U K$ \\ *Corresponding author: sergey.stafeev@gmail.com
}

Received Month X, XXXX; revised Month X, XXXX; accepted Month X, XXXX; posted Month X, XXXX (Doc. ID XXXXX); published Month X, XXXX

\begin{abstract}
Using a binary microlens of diameter $14 \mu \mathrm{m}$ and focal length $532 \mathrm{~nm}(\mathrm{NA}=0.997)$ in resist, we focus a 633-nm laser beam into a near-circular focal spot with dimensions $(0.35 \pm 0.02) \lambda$ and $(0.38 \pm 0.02) \lambda(\lambda$ is incident wavelength) at full-width of half-maximum intensity. The incident light is a mixture of linearly and radially polarized beams generated by reflecting a linearly polarized Gaussian beam at a $100 \mu \mathrm{m} \times 100 \mu \mathrm{m}$ four-sector subwavelength diffractive optical microelement with a gold coating. The focusing of a linearly polarized laser beam (the other conditions being the same) is found to produce an elliptical focal spot measuring $(0.40 \pm 0.02) \lambda$ and $(0.50 \pm 0.02) \lambda$. In both cases, the area of the focal spots is $0.105 \lambda^{2}$. This is the first implementation of subwavelength focusing of light using a pair of microoptic elements (a binary microlens and a micropolarizer). (C) 2012 Optical Society of America

OCIS codes: (050.1380) Binary optics; (050.1965) Diffractive lenses; (050.6624) Subwavelength structures; (180.4243) Nearfield microscopy; (220.4000) Microstructure fabrication; (230.5440) Polarization-selective devices; (260.5430) Polarization. http://dx.doi/org/10.1364/AO.99.099999
\end{abstract}

\section{Introduction}

The manipulation of polarization states of laser light and generation of a prescribed intensity distribution in a given plane by means of subwavelength diffraction gratings was first proposed in Ref. [1]. Subwavelength diffraction gratings were utilized to convert the laser light polarization in Refs. [2-7]. When a linearly polarized light is incident on such a grating, the magnitude of polarization rotation depends on the angle between the incident polarization vector and the direction of the grating lines/grooves. Refs. [2-7] describe gratings operating in transmission and manufactured from a variety of materials for the IR wavelength range. In particular, GaAs gratings for a wavelength of $10.6 \mu \mathrm{m}$ were utilized in Refs. [2-5]. For the grating polarizer, the etch depth was $2.5 \mu \mathrm{m}$. A GaAs grating operating at a wavelength of $1.06 \mu \mathrm{m}$ and characterized by a $240^{-}$ $\mathrm{nm}$ period and 470-nm etch depth was discussed in Ref. [6]. In Ref. [7] an azimuthally polarized laser beam was generated using a subwavelength grating with birefringence properties at $1.55 \mu \mathrm{m}$. The structure was composed of alternating $\mathrm{SiO}_{2}$ and SiN layers of the overall height $8 \mu \mathrm{m}$. It presents a challenging task to manufacture a subwavelength grating in transmission for the visible range due to high aspect ratio (of about 5). Considering this, a reflection four-sector binary grating in a gold film of period $400 \mathrm{~nm}$ and depth $110 \mathrm{~nm}$ was utilized in Ref. [8] to generate a radially polarized light of wavelength $633 \mathrm{~nm}$.

The present authors have reported [9-11] experiments on tightly focusing a linearly polarized laser beam by means of microoptic components. In Ref. [9], the focusing was realized with a binary microaxicon with a circular grating of period $800 \mathrm{~nm}(\mathrm{NA}=0.67)$. In Ref. [10], a binary microlens of focus $f=0.532 \mu \mathrm{m}$, diameter $14 \mu \mathrm{m}$, and microrelief depth $510 \mathrm{~nm}$ was utilized. Using a scanning near-field optical microscope (SNOM), we studied the propagation of a linearly polarized Gaussian beam of wavelength $\lambda=532 \mathrm{~nm}$ through the microlens. A focal spot of size at fullwidth at half-maximum intensity $\mathrm{FWHM}=(0.44 \pm 0.02) \lambda$ was experimentally obtained. By replacing the $532-\mathrm{nm}$ incident wavelength with $\lambda=633 \mathrm{~nm}$, we managed to obtain [11] a tighter focal spot of size $F W H M=(0.40 \pm 0.02) \lambda$ using the same microlens.

Publications concerned with the subwavelength focusing of radially and azimuthally polarized laser beams have also been widely known [12-15]. Focal spots of size $\mathrm{FWHM}=0.43 \mathrm{\lambda}$ $(\mathrm{NA}=0.95)[12,14], \mathrm{FWHM}=0.30 \mathrm{\lambda}(\mathrm{NA}=1.4)[13]$ have been obtained in the numerical and physical experiments. In Ref. [13], the above-mentioned resolution was experimentally attained in recording of 3Tbit of data on a conventional optical disk.

In this work, a 633-nm laser beam was focused using a binary microlens of diameter $14 \mu \mathrm{m}$ and focus $532 \mathrm{~nm}(\mathrm{NA}=0.997)$ fabricated in resist. The beam composed of a mixture of linearly and radially polarized beams, generated by reflection of a linearly polarized Gaussian beam from a gold-coated four-sector subwavelength binary diffractive optical microelement (micropolarizer) of size $100 \times 100 \mu \mathrm{m}$, was focused near the microlens surface into a near-circular focal spot measuring 
$\mathrm{FWHM}=(0.35 \pm 0.02) \lambda$ and FWHM $=(0.38 \pm 0.02) \lambda$. The measurement of the focal spot was conducted with a 20-nm step using a SNOM with a metal cantilever shaped as a hollow pyramid tip with a $70^{\circ}$ vertex angle and a $100-\mathrm{nm}$ pinhole. Such a cantilever is 3 times more sensitive to the transverse electric field component than to the longitudinal field component [16].

\section{Reflection binary micropolarizer}

A gold layer of thickness $160-180-n m$ was deposited on a glass substrate by electron beam evaporation. Then, the golden layer was coated with a layer of electron beam resist (ZEON ZEP520A), and the pattern of the four-sector grating polarizer projected into the resist using a $30-\mathrm{KV}$ electron beam. The sample was then developed in xylene to dissolve resist fragments that had been exposed to the electron beam. The pattern of the grating polarizer was then transferred into the gold layer using by sputtering with an Argon plasma.. In the final stage, the remaining resist was removed with an oxygen plasma, resulting in a grating polarizer pattern engraved in gold. The duration of the reactive ion etching process was optimized so as to achieve the etch depth of the gold of about $110 \mathrm{~nm}$.

Figure 1a depicts a SEM image of the grating polarizer central fragment. Note that the overall size of the polarizer is $100 \times 100 \mu \mathrm{m}$. and the fill factor was equal to 0.5 . The quality of the micropolarizer was superior to that of Ref. [8].
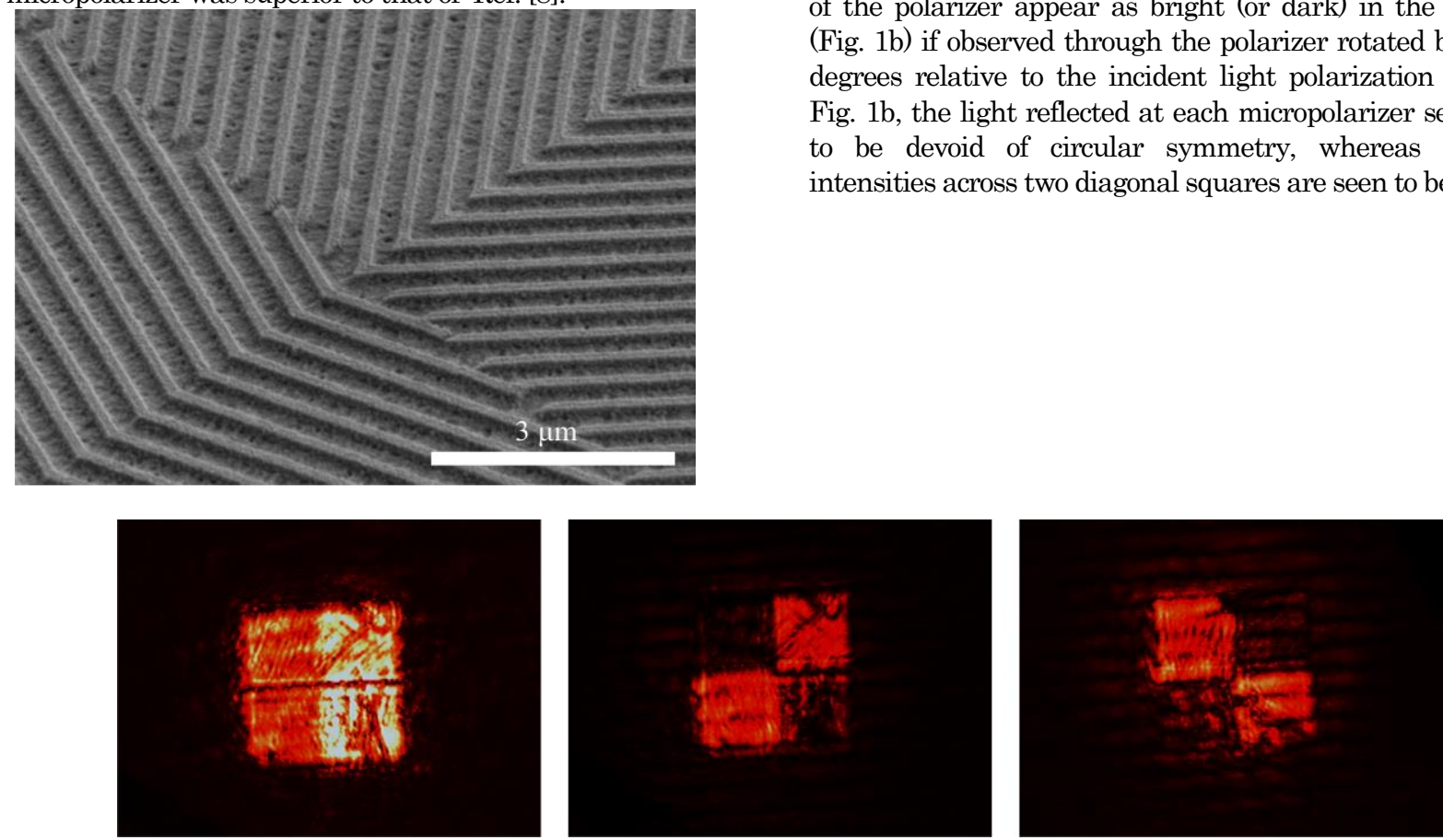

Fig. 2. Image of a 100x100- $\mu \mathrm{m}$ four-sector micropolarizer (Fig.1a) obtained in reflection the 633-nm laser light for the differently directed axis of the output polarizer (put before a CCD-camera): $0^{\circ}(\mathrm{a}), 45^{\circ}(\mathrm{b}),-45^{\circ}(\mathrm{c})$.

Figure 2a shows an image of the four-sector micropolarizer of Fig. 1a obtained using an output polarizer with its axis directed vertically, considering that the incident light is linearly polarized in the horizontal plane. In this case, all four sectors of the micropolarizer turn out to be bright (Fig. 1a). Note that two right sectors of the micropolarizer have a higher reflectance when compared with the two left ones. Figure $2 \mathrm{~b}$ shows a micropolarizer image obtained when the axis of the polarizer placed in reflected light is rotated by $45^{\circ}$ relative to the horizontal

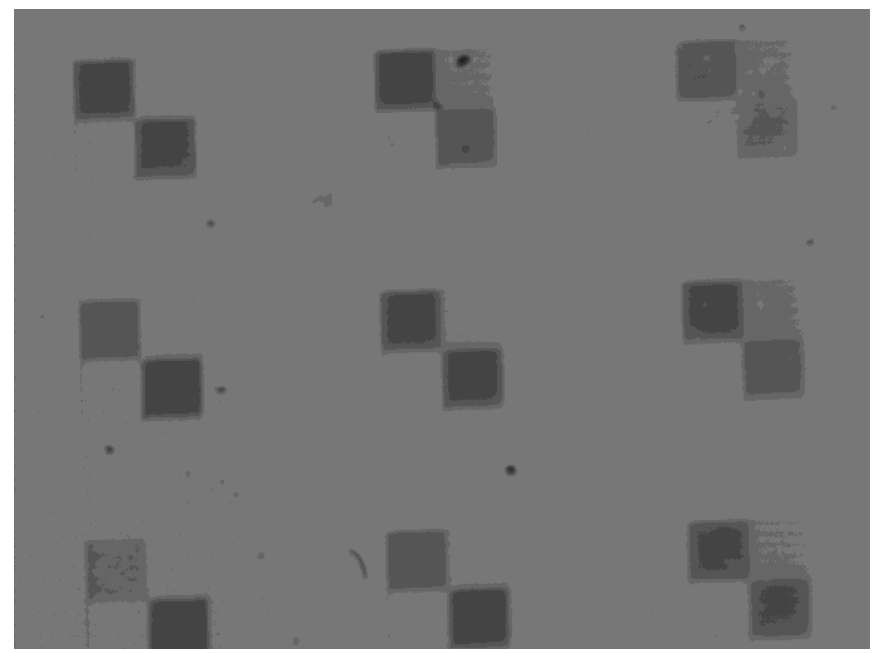

Fig. 1. (a) SEM image of the central $13 \times 13-\mu \mathrm{m}$ fragment of a golden binary four-sector grating polarizer: period- $400 \mathrm{~nm}$, groove depth- $110 \pm 10 \mathrm{~nm}$ and (b) the image of a golden substrate comprising nine micropolarizer prototypes was obtained in linearly polarized white light and observed through a polarizer rotated by $45^{\circ}$. Each dark square measures 50x50- $\mu \mathrm{m}$.

When the linearly polarized white light is reflected at the micropolarizer under study (Fig. 1a) just two of the four sections of the polarizer appear as bright (or dark) in the image plane (Fig. 1b) if observed through the polarizer rotated by +45 or -45 degrees relative to the incident light polarization plane. From Fig. 1b, the light reflected at each micropolarizer section is seen to be devoid of circular symmetry, whereas the average intensities across two diagonal squares are seen to be different. axis. Figure 2c shows the same pattern but with the output polarizer axis rotated by $-45^{\circ}$ relative to the horizontal axis. In this case, two of four micropolarizer's sectors found on the diagonals are observed as bright.

Unlike a perfect radially polarized beam that generates a ringlike intensity pattern, in our case, the lack of circular symmetry in the far field results in the micropolarizer generating a beam with a square-like transverse intensity pattern (Fig. 3a) with its intensity maxima (Figs. 3b and 3c) found on the corresponding 
diagonals (as in Fig. 2b,c). When the output polarizer is rotated by $+45^{\circ}$ and $-45^{\circ}$,the resulting beam is a mixture of linearly and radially polarized beams.
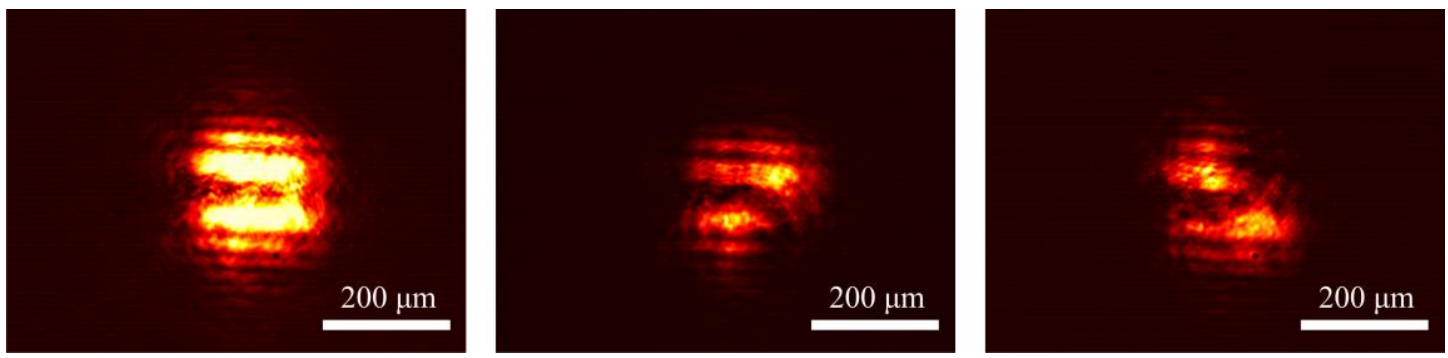

Fig.3 Far-field intensity patterns for the laser light $(633 \mathrm{~nm})$ reflected at the micropolarizer (Fig. 1): (a) without an output polarizer, with a polarizer rotated by (b) $45^{\circ}$ and (c) $-45^{\circ}$

\section{Binary microlens in a resist}

A high quality binary microlens $[10,11]$ was fabricated in ZEP520A resist (refractive index $n=1.52$ ) by electron beam lithography. Figure 4 depicts a SEM image of the microlens: mirorelief depth- $510 \mathrm{~nm}$, diameter- $14 \mu \mathrm{m}$, outermost zone- $0.5 \mathrm{\lambda}$ $=266 \mathrm{~nm}$. The microlens is composed of 12 rings and a central disk.

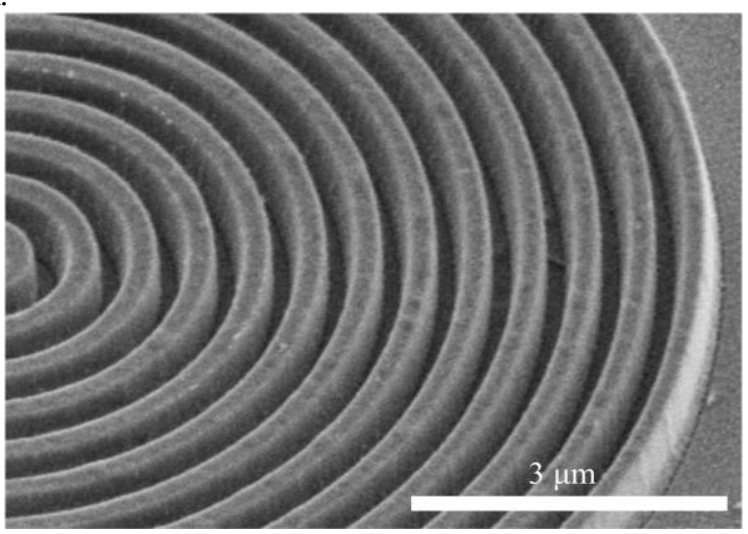

Fig. 4. SEM image of the microlens with a 18000x magnification.

The radii of the microlens rings were calculated using the familiar formula $r_{m}=\left(m \lambda f+m^{2} \lambda^{2} / 4\right)^{3 / 2}$, where $f=\lambda=532 \mathrm{~nm}$ is the focal length and $m$ is the radius number. The microlens has $\mathrm{NA}=0.997$. When the microlens in Fig. 4 is illuminated by a linearly polarized Gaussian beam of wavelength $633 \mathrm{~nm}$ and radius approximately equal to that of the microlens $(7 \mu \mathrm{m})$, the focal spot is formed nearer than it is suggested by the calculations $(532 \mathrm{~nm})$, being found 230-nm away from the microlens, as shown in Ref. [11]. Using the FDTD method [11], the numerically calculated minimal and maximal dimensions of the focal spot were shown to equal $\mathrm{FWHM}_{\min }=(0.40 \pm 0.02) \lambda$ and $\mathrm{FWHM}_{\max }=(0.87 \pm 0.02) \lambda$. In the meantime, the minimal and maximal dimensions of the focal spot experimentally measured with a SNOM were $\mathrm{FWHM}_{\text {min }}=(0.40 \pm 0.02) \lambda$ and $\mathrm{FWHM}_{\max }=$ $(0.60 \pm 0.02) \lambda$.

\section{Simulation}

Using the FDTD-method, the focusing of a radially polarized RTEM $_{01}$ mode of wavelength $\lambda=633 \mathrm{~nm}$ and mode parameter $R=10 \lambda$ by means of a microlens of focal length $532 \mathrm{~nm}$ intended to operate at a wavelength of $532 \mathrm{~nm}$ was simulated. In Fig. 5 is (a) the intensity profile in the focal point is shown and (b) the expected experimental profile that would be measured using a SNOM with a pyramid metal cantilever [16], In Fig. 5a, the focal spot size is FWHM $=0.37 \lambda$.
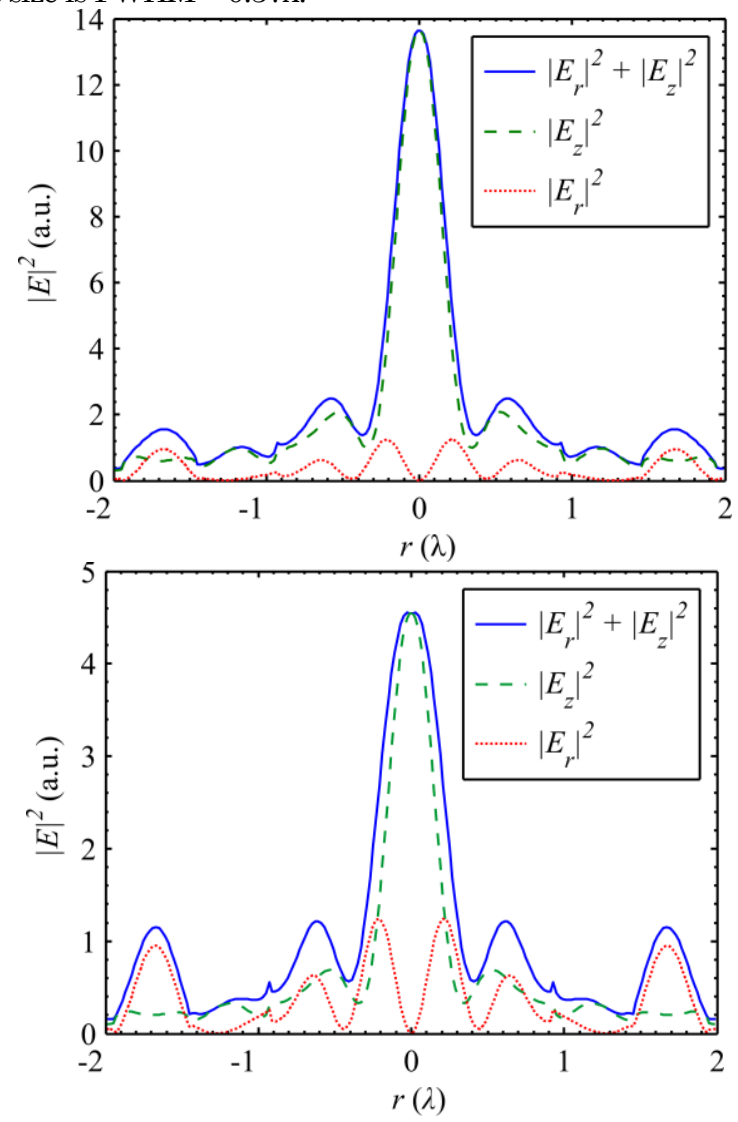

Fig. 5. (a) Intensity profile in the focus near the microlens surface when focusing the $\mathrm{R}-\mathrm{TEM} \mathrm{M}_{01}$ mode with the parameter $\mathrm{R}=10 \mathrm{\lambda}(\mathrm{a})$ и and (b) the same NSOM-aided pattern 3 times less sensitive to the longitudinal component.

Figure 5 suggests that should there be a perfect radially polarized binary-microlens-aided focus (Fig. 4), the SNOM image of the focal spot would appear as a circle (Fig. 5b) of size $\mathrm{FWHM}=0.47 \lambda$. That is to say that the image of a tight focus of a perfect radially polarized beam obtained with a SNOM with a hollow metal pyramid tip will be larger than the real spot. In the case under study, instead of the real size of FWHM $=0.37 \lambda$ the SNOM image is supposed to be of size FWHM $=0.47 \lambda$. 
However, the four-sector micropolarizer in Fig. 1a generates a mixed linearly and radially polarized beam [8]. Below, we simulate in which way this type of beam will be focused by the microlens of Fig. 4 and in which way the focal spot is to be imaged by the SNOM. Figure 6 shows the intensity pattern generated $200^{-} \mu \mathrm{m}$ apart from the micropolarizer of Fig. 1a, with the arrows showing the polarization directions.

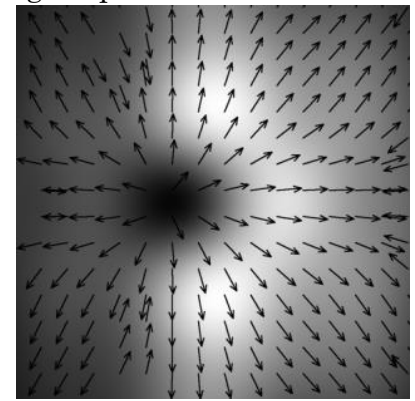

Fig. 6. Numerically modeled intensity pattern $|\mathrm{E}|^{2} 200 \mu \mathrm{m}$ apart from the micropolarizer of Fig. 1a. The frame size is $(5 \times 5) \mu \mathrm{m}$.
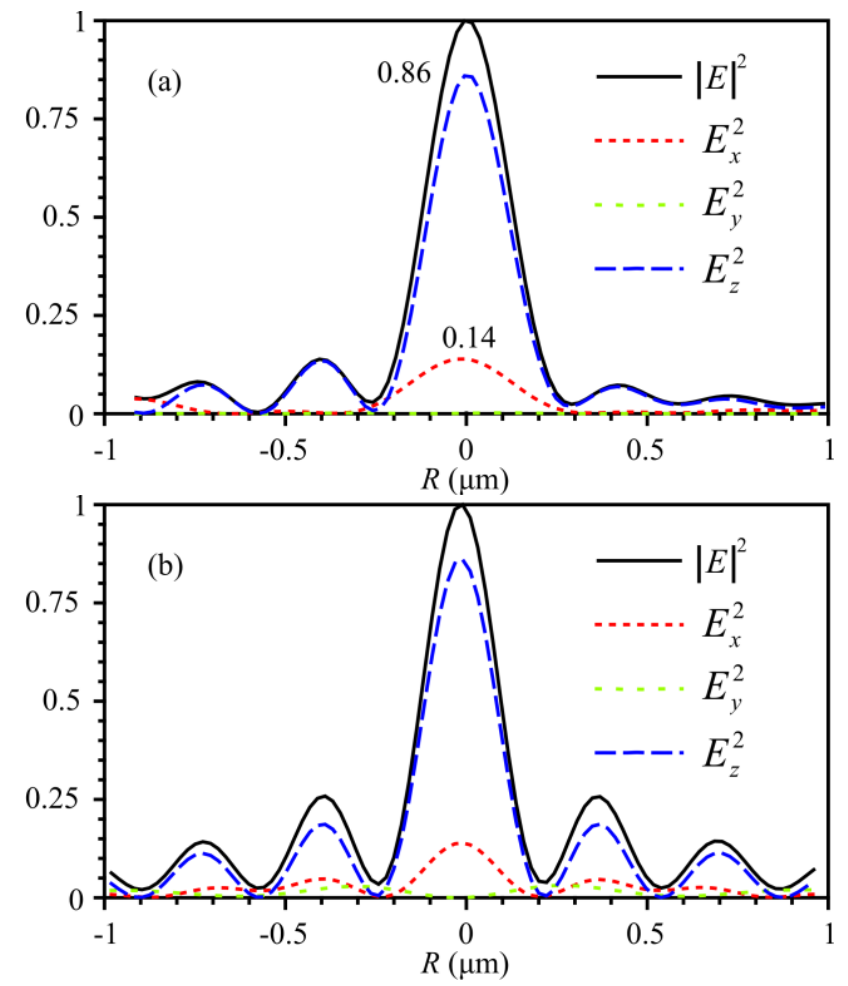

Fig. 7. FDTD-method: Intensity profiles of the electric field components in the focus of the microlens of Fig. 4 along the (a) $\mathrm{X}^{-}$and (b) Y-axes.

Figure 7 shows the intensity profiles in the focus of the microlens in Fig. 4, which is illuminated by a mixed linearly and radially polarized beam (Fig. 6) from the micropolarizer in Fig. 1a. From Fig. 7, the transverse intensity component is seen to be characteristic of the linearly polarized light, with the longitudinal component corresponding to the radially polarized light. This has prompted us to define the beam under study as having a mixed, linear and radial polarization. In this case, instead of being a perfect circle (see a perfect radial polarization in Fig. 5), the focal spot is elliptical with the axial dimensions FWHM $_{\max }=$ $(0.40 \pm 0.02) \lambda$ (Fig.7a) and $F_{W H M}$ min $=(0.35 \pm 0.02) \lambda$ (Fig.7b). Let us recall that a circular focal spot for perfect radial polarization had been found to have an interim size of FWHM $=0.37 \mathrm{~A}$ (Fig. 5a). In terms of their area, the difference between these focal spots is as low as $3 \%$. From Fig. 7, the transverse intensity $E_{x}^{2}$ is seen not to take a zero value on the optical axis, taking a maximum value instead, although the longitudinal intensity $E_{Z}^{2}$ is 6 times the transverse intensity component $E_{X}^{2}$. Figure 8 depicts intensity profiles similar to those shown in Fig. $7 \mathrm{~b}$ for the three-times decreased longitudinal intensity. In this case, the focal spot is seen to be somewhat larger in size: $\mathrm{FWHM}_{\min }=$ (0.36 \pm 0.02$) \lambda$ (Fig. 8). A comparison of Figs. 5 and 8 suggests that for both radial and mixed, linear-radial polarization, SNOMaided measurements with a metal hollow pyramid cantilever [16], (3-times more sensitive to the transverse than to the longitudinal electric field component), give an intensity maximum at the focal spot center.

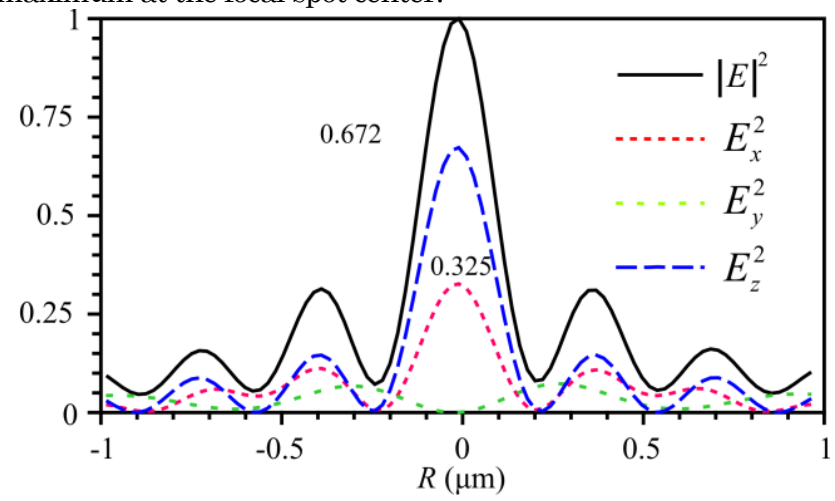

Fig.8. FDTD-method: Intensity profiles for the electric field components in the focus of the microlens in Fig. 4 on the Y-axis. The longitudinal intensity $\mathrm{E}_{z}{ }^{2}$ has been decreased by a factor of 3 .

In Section 5, we show that a mixed, linearly and radially polarized beam from the micropolarizer of Fig. 1a can be focused by means of the microlens of Fig. 4 into a near-circular focal spot, which is tighter than that reported in Ref. [11].

\section{Experimental focusing of a mixed linear-radially polarized beam with a binary microlens}

The experiment studied the tight focusing of an optical beam reflected from a micropolarizer (Fig. 1a) that had converted the linear polarization into the radial one. The experimental optical setup is shown in Fig. 9. 


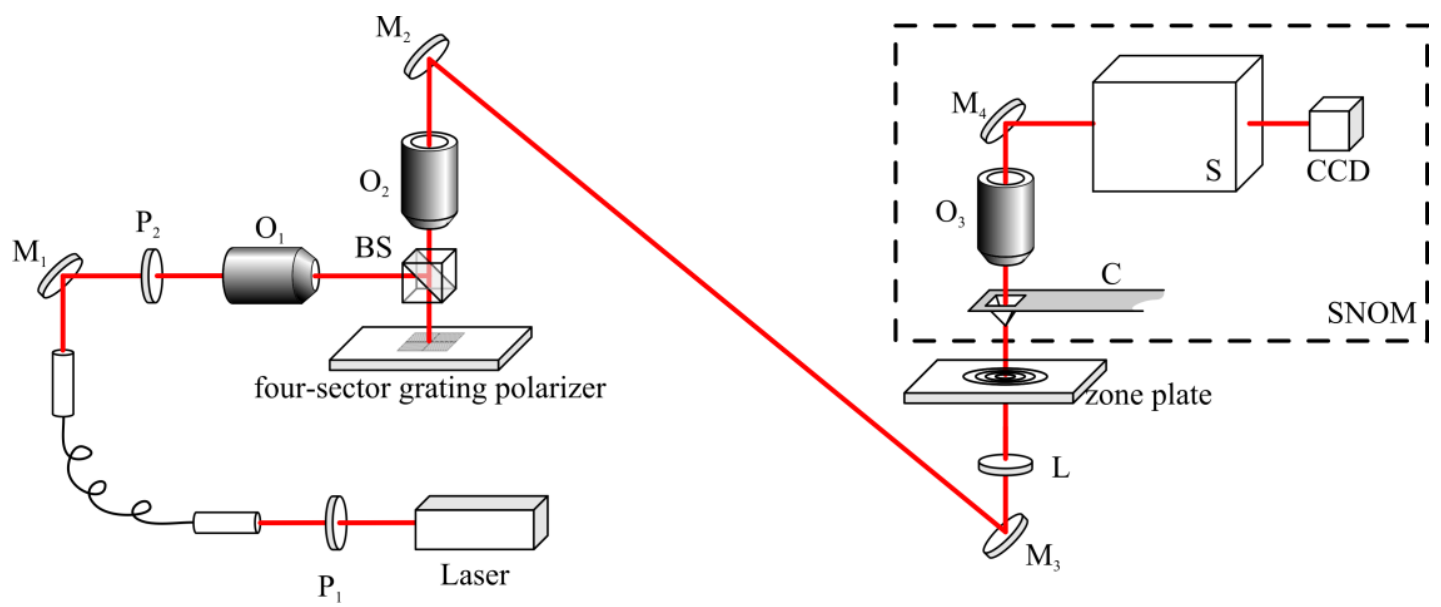

Fig. 9. Experimental setup. Laser- a He-Ne laser $(\lambda=633 \mathrm{~nm}), \mathrm{P}_{1}, \mathrm{P}_{2^{-}}$polarizers, $\mathrm{M}_{1}, \mathrm{M}_{2}, \mathrm{M}_{3}, \mathrm{M}_{4}^{-}$mirrors, $\mathrm{O}_{1^{-}}$3.7x objective, $\mathrm{BS}^{-}$light splitting cube, $\mathrm{O}_{2}{ }^{-}$ $20 \mathrm{x}$ objective, $\mathrm{O}_{3}-100 \mathrm{x}$ objective, C- cantilever, $\mathrm{L}$ - lens, $\mathrm{S}^{-}$spectrometer, CCD - CCD-camera.

The light from a He-Ne laser $(\lambda=633 \mathrm{~nm})$ was focused with an objective $\mathrm{O}_{1}$ onto a four-sector micropolarizer (Fig. 1a), which converted the linear polarization into the mixed, linear-radial one. The polarizers $\mathrm{P}_{1}$ and $\mathrm{P}_{2}$ served the double purpose of verifying that the light incident on the micropolarizer was linearly polarized and attenuating the light power. After reflecting at the micropolarizer, the beam was focused onto the bottom of the microlens of Fig. 4 of focal length $532 \mathrm{~nm}$, producing a subwavelength focal spot after passing it. The intensity pattern of the focal spot was measured with a SNOM Ntegra Spectra (NT-MDT) (shown in a dashed line rectangle in Fig. 9). Figure 10a illustrates a SNOM-aided intensity pattern, with the minimal and maximal size of the focal spot measuring $\mathrm{FWHM}_{\max }=(0.38 \pm 0.02) \lambda$ (Fig. $\left.10 \mathrm{~b}\right)$ and $\mathrm{FWHM}_{\min }=(0.35 \pm 0.02) \mathrm{\lambda}$ (Fig.10c). The simulation results for this case are shown in Fig. 10 (black curve): with the focal spot measuring $\mathrm{FWHM}_{\max }=$ $(0.40 \pm 0.02) \lambda$ (Fig.10b) and $\mathrm{FWHM}_{\min }=(0.36 \pm 0.02) \lambda$ (Fig.10c). Thus, the discrepancy between the experiment and simulation falls within the RMS error $2 \%$. In the focal spot, the side-lobes of the diffraction pattern amount to $30 \%$ of the major intensity peak (Fig. 10c). Thus, one can infer that a high-NA binary microlens (Fig.4) operates as a binary axicon, generating a focal spot with the intensity profile described by the squared zero-order Bessel function. For a Bessel beam, the diffraction limit is known to equal FWHM $=0.36 \lambda$.

To evaluate the effect of the micropolarizer, the substrate containing the micropolarizer was shifted in the transverse direction to allow the light to be reflected at the relief-free golden surface. This resulted in a microlens-aided focal spot of dimensions $\mathrm{FWHM}_{\max }=(0.50 \pm 0.02) \lambda$ and $\mathrm{FWHM}_{\min }=$ $(0.40 \pm 0.02) \lambda$. Thus, when focusing a linearly polarized beam, the resulting focal spot was found to be more elliptical

$\mathrm{S}_{\text {linear }}=\Pi\left(\mathrm{FWHM}_{\min } \mathrm{FWHM}_{\max }\right) / 4=0.157 \lambda^{2}$

$\mathrm{S}_{\text {radial }}=\Pi\left(\mathrm{FWHM}_{\min } \mathrm{FWHM}_{\max }\right) / 4=0.105 \lambda^{2}$
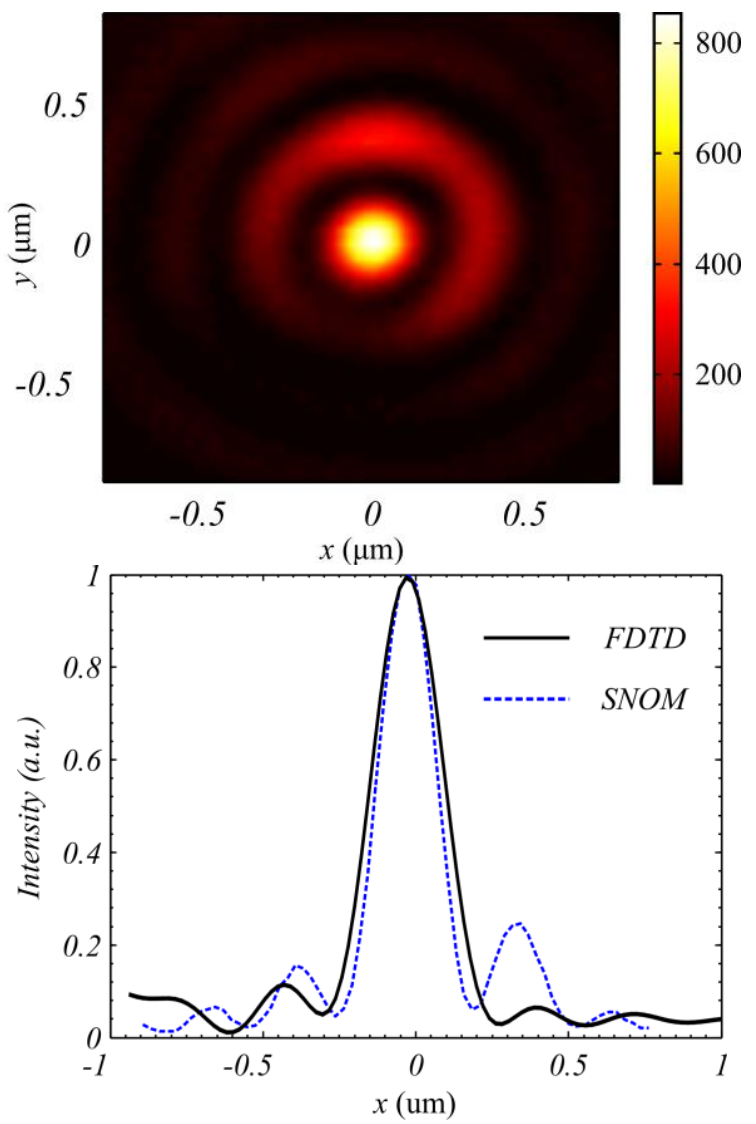


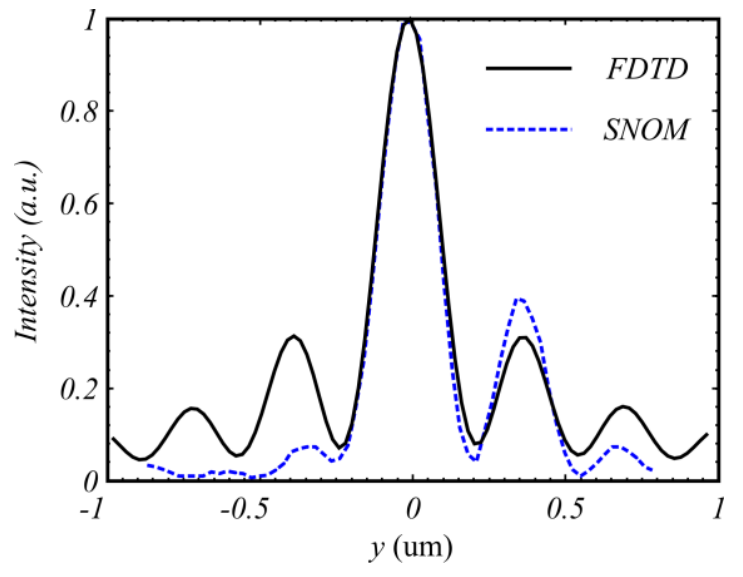

Fig.10. An illustration of a SNOM-aided intensity distribution when a beam reflected at the micropolarizer is focused by the microlens: (a) Twodimensional intensity pattern and $(b, c)$ intensity profiles along the perpendicular axes.

\section{Conclusion}

Using a binary microlens of diameter $14 \mu \mathrm{m}$ and focal length 532 $\mathrm{nm}(\mathrm{NA}=0.997)$ we have focused a 633-nm laser beam into a near-circular focal spot with dimensions $(0.35 \pm 0.02) \lambda$ and $(0.38 \pm 0.02) \lambda$ at full-width of half-maximum intensity. The incident light is a mixture of linearly and radially polarized beams generated by reflecting a linearly polarized Gaussian beam at a gold $100 \mu \mathrm{m}$ x $100 \mu \mathrm{m}$ four-sector subwavelength diffractive optical microelement, with the sector gratings of period $400 \mathrm{~nm}$ and microrelief depth $110 \mathrm{~nm}$. The focusing of a linearly polarized laser beam (other conditions being the same) has been found to produce an elliptical focal spot measuring $(0.40 \pm 0.02) \lambda$ and $(0.50 \pm 0.02) \lambda$. In both cases, the area of the focal spots is $0.105 \lambda^{2}$. The subwavelength focusing of light using a pair of microoptic elements (a binary microlens and a micropolarizer) has been implemented for the first time. It is notable that recently [17] a focal spot of size $0.25 \lambda$ has been attained by focusing an azimuthally polarized first-order vortex beam by means of a conventional immersion microlens with $\mathrm{NA}=1.4$. By reducing this focal spot and the one reported in this work, equal to $0.35 \lambda$, to the same NA, we obtain the same-size focal spots, namely,

$\mathrm{FWHM}=0.25 \lambda=0.25 \lambda^{*} 1.4 / \mathrm{NA}=0.35 \mathrm{\lambda} / \mathrm{NA}$,

$\mathrm{FWHM}=0.35 \lambda=0.35 \lambda^{*} 0.997 / \mathrm{NA}=0.35 \lambda / \mathrm{NA}$.

From formulae above it follows that immersion microlens (fig. 4) gives the same result as in [17].

\section{Acknowledgements}

This work was partially funded by the Ministry of Education and Science of the Russian Federation and Russian Federation Presidential grants for support of leading scientific schools (NSh3970.2014.9), Young Candidate of Science grant (MK4816.2014.2), and Russian Foundation for Basic Research grants \#14-07-31092, 14-07-97039, 14-29-07133, 15-07-01174 and European Research Council Starting grant (no. 337508)

\section{References}

1. V.V. Kotlyar and O.K. Zalyalov, "Design of diffractive optical elements modulating polarization," Optik 103, 125130 (1996).
2. Z. Bomzon, V. Kleiner, and E. Hasman, "Pancharatnam-Berry phase in space-variant polarization-state manipulations with subwavelength gratings," Opt. Lett. 26, 1424-1426 (2001)

3. Z. Bomzon, G. Biener, V. Kleiner, and E. Hasman, "Radially and azimuthally polarized beams generated by space-variant dielectric subwavelength gratings," Opt. Lett. 27, 285-287 (2002)

4. A. Niv, G. Biener, V. Kleiner, and E. Hasman, "Formation of linearly polarized light with axial symmetry by use of space-variant subwavelength gratings," Opt. Lett. 28, 510512 (2003).

5. U. Levy, C.-H. Tsai, L. Pang, and Y. Fainman, "Engineering space-variant inhomogeneous media for polarization control," Opt. Lett. 29, 1718-1720 (2004)

6. G.M. Lerman and U. Levy, "Generation of a radially polarized light beam using space-variant subwavelength gratings at $1064 \mathrm{~nm}$," Opt. Lett. 33, 2782-2784 (2008)

7. A. Mehta, J.D. Brown, P. Srinivasan, R.C. Rumpf, and E.G. Johnson, "Spatially polarizing autocloned elements," Opt. Lett. 32, 1935-1937 (2007)

8. A.G. Nalimov, L. O'Faolain, S.S. Stafeev, M.I. Shanina, and V.V.Kotlyar, "Reflected four-sectors subwavelenghth mictooptics element for polarization conversion from linear to radial," Computer Optics 38, 229-236 (2014).

9. V.V. Kotlyar, S.S. Stafeev, L. O'Faolain, and V.A. Soifer, "Tight focusing with a binary microaxicon," Opt. Lett. 36, 3100-3102 (2011)

10. S.S. Stafeev, L. O'Faolain, M.I. Shanina, V.V. Kotlyar, and V.A. Soifer, "Subwavelength focusing using Fresnel zone plate with focal length of 532nm," Computer Optics 35, 460-461 (2011).

11. V.V. Kotlyar, S.S. Stafeev, Y. Liu, L. O'Faolain, and A.A. Kovalev, "Analysis of the shape of a subwavelength focal spot for the linearly polarized light," Appl. Opt. 52, 330-339 (2013)

12. G.H. Yuan, S.B. Wei, and X.-C. Yuan, "Nondiffracting transversally polarized beam," Opt. Lett. 36, 3479-3481 (2011)

13. X. Li, Y. Cao, and M. Gu, "Superresolution-focal-volume induced 3.0 Tbytes/disk capacity by focusing a radially polarized beam," Opt. Lett. 36, 2510-2512 (2011)

14. J. Lin, K. Yin, Y. Li, and J. Tan, "Achievement of longitudinally polarized focusing with long focal depth by amplitude modulation," Opt. Lett. 36, 1185-1187 (2011)

15. H. Lin, B. Jia, and M. Gu, "Generation of an axially superresolved quasi-spherical focal spot using an amplitudemodulated radially polarized beam," Opt. Lett. 36, 24712473 (2011)

16. S.S. Stafeev and V.V. Kotlyar, "Special aspects of subwavelength focal spot measurement using near-field optical microscope," Computer Optics 37, 332-340 (2013).

17. X. Li, P. Venugopalan, H. Ren, M. Hong, and M. Gu, "Super-resolved pure-transverse focal fields with an enhanced energy density through focus of an azimuthally polarized first-order vortex beam," Opt. Lett. 39, 5961-5964 (2014) 